\title{
Endokrin resistentes Mammakarzinom: Lebensqualität erhalten
}

In der PALOMA-3-Studie hatte Fulvestrant plus Palbociclib das progressionsfreie Überleben im Vergleich zu Fulvestrant plus Placebo bei hormonrezeptorpositivem, HER2-negativem, endokrin resistentem metastasiertem Brustkrebs verbessert. Nun ging es um die Lebensqualität der Patientinnen.

n dieser Phase-III-Studie waren 521 Patientinnen mit Fulvestrant plus entweder Palbociclib oder Placebo behandelt worden und hatten ihre Lebensqualität mithilfe des Fragebogens EORTC QLQC30 und dem Brustkrebsmodul QLQ-

BR23 dokumentiert. Bis zum 14. Zyklus hatten mehr als $95 \%$ der Patientinnen jeder Gruppe mindestens eine Frage beantwortet. Während der Therapie fielen die Scores für globale Lebensqualität zugunsten der Kombination aus (66,1 vs.

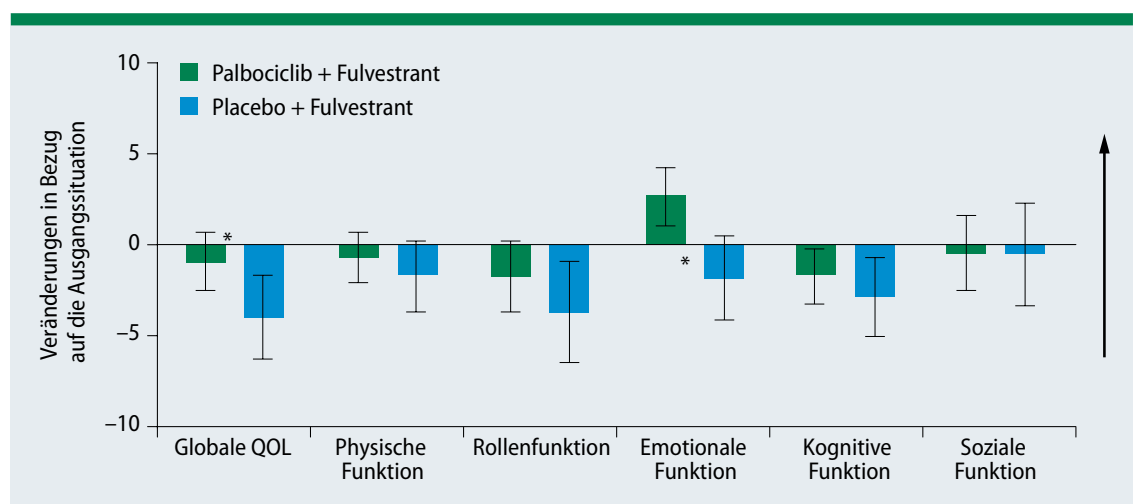

Abb. 1: Veränderung der EORTC QLQ-C30-Scores (globale Lebensqualität und funktionelle Domänen).

\section{Metastasiertes Mammakarzinom: Erhaltung mit Aromatasehemmer ohne Nutzen}

In der AROBASE-Studie wurde der Nutzen einer endokrinen Therapie zusätzlich zur Fortführung einer Bevacizumab-Therapie in der Erhaltungstherapie beim Östrogenrezeptor(ER)-positiven metastasierten Mammakarzinom untersucht.

n dieser Phase-III-Studie wurden Patientinnen mit einer nach 16-24-wöchiger Therapie mit einem Taxan plus Bevacizumab nicht fortschreitender Erkrankung in zwei Arme randomisiert: Sie erhieltenentwederweiterhindieTaxan-plus Bevacizumab-Therapie oder eine Erhaltungstherapie mit Bevacizumab plus Exemestan. Primärer Endpunkt war das progressionsfreie Überleben (PFS).

Eine Zwischenauswertung der ersten 98 Patienten zeigte, dass die Wahrscheinlichkeit, eine signifikante Verbes- serung des PFS zu erreichen, nur $7 \%$ betrug. Das 6-Monats-PFS betrug zu diesem Zeitpunkt 70,1\% unter Taxan plus Bevacizumab und $54 \%$ unter Exemestan plus Bevacizumab. Dies führte zu der Empfehlung, keine weiteren Patienten mehr in die Studie aufzunehmen.

Die 6-Monats-PFS-Raten lagen bei $67,2 \%$ unter Taxan plus Bevacizumab und bei $55,2 \%$ unter Exemestan plus Bevacizumab (Hazard Ratio [HR] 1,0; $\mathrm{p}=0,998$ ), die Medianwerte bei 12,5 bzw. 12,3 Monaten. Auch in der Lebensquali-
63,0\%, p = 0,0313) (Abb. 1). Zudem verzögerte die Addition von Palbociclib signifikant die Verschlechterung dieses Parameters $(p=0,025)$. Während es in den funktionellen Domänen des QLQC30 keine signifikanten Unterschiede zwischen den Gruppen gab, wurden in der Palbociclib-Gruppe aber Schmerzen signifikant besser $(-3,3$ vs. 2,$0 ; p=0,0011)$, und Übelkeit/Erbrechen beeinträchtigten die Patienten weniger (1,7 vs. 4,2 ; $\mathrm{p}=0,0369$ ). Die mediane Zeit bis zur Verschlechterung von Schmerzen betrug 8 Monate im Palbociclib- und 2,8 Monate im Placeboarm (Hazard Ratio 0,642, $\mathrm{p}<0,001)$.

Fazit: Die Therapie mit Fulvestrant plus Palbociclib konnte eine gute Lebensqualität bei Patientinnen mit endokrin resistentem Mammakarzinom aufrechterhalten - bei deutlich verzögerter Krankheitsprogression.

Judith Neumaier

Harbeck $N$ et al. Quality of life with palbociclib plus fulvestrant in previously treated hormone receptor-positive, HER2-negative metastatic breast cancer: patient-reported outcomes from the PALOMA-3 trial. Ann Oncol. 2016;27(6):104754.

tät ergaben sich keine Unterschiede zwischen den Gruppen. Im Taxan-Arm wurde die Taxan-Gabe bei $94,9 \%$ der Patientinnen vorzeitig beendet, hauptsächlich wegen Toxizitäten (49,2\%). Aktualisierte Daten nach median 35 Monaten zeigten weiterhin keine Unterschiede zwischen den Gruppen. Die Sterberaten betrugen $44 \%$ bzw. $55 \%$.

Fazit: Diese Ergebnisse sprechen nicht für die Erhaltungstherapie mit Exemestan plus Bevacizumab bei ER-positivem, HER2-negativem, metastasiertem Mammakarzinom, das nach Erstlinientherapie mit einem Taxan und Bevacizumab nicht progredient ist. Judith Neumaier

Trédan $\mathrm{O}$ et al. A phase III trial of exemestane plus bevacizumab maintenance therapy in patients with metastatic breast cancer after firstline taxane and bevacizumab: a GINECO group study. Ann Oncol. 2016;27(6):1020-9. 